\title{
Effects of dynamical masses of gluons and quarks on hadronic B decays
}

\author{
C. M. Zanetti* and A. A. Natale ${ }^{\dagger}$ \\ * Instituto de Física, Universidade de São Paulo, São Paulo, SP, Brazil \\ ${ }^{\dagger}$ Instituto de Física Teórica, São Paulo State University, São Paulo, SP, Brazil
}

\begin{abstract}
.
We study hadronic annihilation decays of $B$ mesons within the perturbative QCD at collinear approximation. The regulation of endpoint divergences is performed with the help of an infrared finite gluon propagator characterized by a non-perturbative dynamical gluon mass. The divergences at twist- 3 are regulated by a dynamical quark mass. Our results fit quite well the existent data of $B^{0} \rightarrow D_{s}^{-} K^{+}$and $B^{0} \rightarrow D_{s}^{-*} \bar{K}^{+}$for the expected range of dynamical gluon masses. We also make predictions for the rare decays $B^{0} \rightarrow K^{-} K^{+}, B_{s}^{0} \rightarrow \pi^{-} \pi^{+}, \pi^{0} \pi^{0}, B^{+} \rightarrow D_{s}^{(*)+} \bar{K}^{0}, B^{0} \rightarrow D_{s}^{ \pm(*)} K^{\mp}$ and $B_{s}^{0} \rightarrow D^{ \pm(*)} \pi^{\mp}, D^{0} \pi^{0}$.
\end{abstract}

Keywords: B meson; hadronic decays; infrared cutoff.

PACS: $12.38 . \mathrm{Bx} ; 12.38 . \mathrm{Aw} ; 12.38 . \mathrm{Lg} ; 13.25 . \mathrm{Hw}$

\section{INTRODUCTION}

Perturbative QCD has been largely applied in the study of $B$ meson decays, specially in non-leptonic decays which requires the calculation of hadronic matrix elements. In such processes the long distance interactions between the initial and final state mesons impose a difficult obstacle in amplitudes calculations. In the pQCD approach the calculation of the matrix elements can be performed due to the large scale involved in the strong interactions $\left(\mathscr{O}\left(m_{B}\right)\right)$. The perturbative dynamics can be factorized from the nonperturbative dynamics by applying the hard exclusive scattering Brodsky and Lepage's approach [1]. However, in the collinear approximation of pQCD, the amplitude calculation of non-factorizable diagrams (spectator interaction and annihilation) presents end-point divergences that can not be absorbed into the mesons wave functions to preserve the factorization as usual [2]. Although these contributions in general are power suppressed and are negligible in front of the factorizable contributions, it is important to have a reliable calculation of the annihilation diagrams due to the strong phases that are relevant in the determination of the $\mathrm{CP}$ violation parameters, and also in the evaluation of pure annihilation processes. These divergences can be treated by different approaches, for example [2,3]. In this work, we present a study of the regulation of the divergences using the prescription of an IR finite gluon propagator, which appears as a non-perturbative solution of the gluonic Schwinger-Dyson equations (SDE) [4]. For consistency with the gluon dressing and in order to deal with the collinear divergences that appear at twist-3, we will also use the dynamical masses of the light quarks. We focus on pure annihilation decays, in which the dependence on the regulation will be more evident. 


\section{PERTURBATIVE QCD APPROACH}

Weak decays of $B$ mesons are described by an effective Hamiltonian at a renormalization scale $\mu \ll M_{W}$. This effective Hamiltonian is formally obtained using the Operator Product Expansion (OPE), describing effective interactions through four-quark operators [8]. The amplitude of the decay $B \rightarrow f$ is obtained from:

$$
\mathscr{A}(B \rightarrow f)=\left\langle f\left|\mathscr{H}_{\mathrm{eff}}\right| B\right\rangle=\frac{G_{F}}{\sqrt{2}} \sum_{i} V_{\mathrm{CKM}}^{i} C_{i}(\mu)\left\langle Q_{i}\right\rangle,
$$

where $G_{F}$ is the Fermi constant, $V_{\mathrm{CKM}}$ are Cabibbo-Kobayashi-Maskawa factors, $Q_{i}$ are the four-quarks operators contributing to the decay and $C_{i}(\mu)$ are the Wilson coefficients; $\left\langle Q_{i}\right\rangle=\left\langle f\left|Q_{i}\right| B\right\rangle$ are the hadronic matrix elements between the initial and final states.

In the pQCD formalism the hadronic matrix elements are obtained through a convolution of the hard scattering kernel and the distribution amplitudes of the mesons involved in the process. In the case of a two-body non-leptonic decay $B \rightarrow M_{1} M_{2}$ in the collinear approach, the matrix element of a given operator $Q_{i}$ is [1]:

$$
\left\langle Q_{i}\right\rangle=\int \mathrm{d} x \mathrm{~d} y \mathrm{~d} z T_{i}(x, y, z) \Phi_{M_{1}}(x) \Phi_{M_{2}}(y) \Phi_{B}(z),
$$

where $x, y, z$ are the momentum fractions, $\Phi_{M}(x)$ are the light-cone distribution amplitudes for the quark-antiquark states of the mesons decomposed in terms of spin structure, which are non-perturbative functions of the momentum fraction carried by the partons; $T_{i}$ is the hard scattering kernel that can be perturbatively computed as function of the light-cone momenta of collinear partons.

\section{REGULATION OF DIVERGENCES}

In this work we deal with the soft divergences due to the IR vanishing gluon using the non-perturbative propagator proposed by Cornwall [4]:

$$
D_{g}\left(Q^{2}\right)=\frac{1}{Q^{2}+M_{g}\left(Q^{2}\right)}, \quad M_{g}^{2}\left(Q^{2}\right)=m_{g}^{2}\left[\ln \left(\frac{Q^{2}+4 m_{g}^{2}}{\Lambda^{2}}\right) / \ln \left(\frac{4 m_{g}^{2}}{\Lambda^{2}}\right)\right]^{-\frac{12}{11}},
$$

where $m_{g}=500 \pm 200 \mathrm{MeV}$ is the dynamical gluon mass scale. A simple fit for the coupling constant that is factored out in this procedure is given by [4]

$$
\bar{\alpha}_{s d}\left(q^{2}\right)=\frac{1}{4 \pi b \ln \left[\left(4 m_{g}^{2}-q^{2}-\imath \varepsilon\right) / \Lambda^{2}\right]},
$$

where $b=\left(33-2 n_{f}\right) / 48 \pi^{2}$. This prescription for the gluon propagator has already been successfully used in previously works (se e.g. [5], and references therein).

For the collinear divergences we use the dressed quark propagator that can be written as

$$
S(p)=\frac{Z\left(p^{2}\right)}{l \not p+M\left(p^{2}\right)}
$$




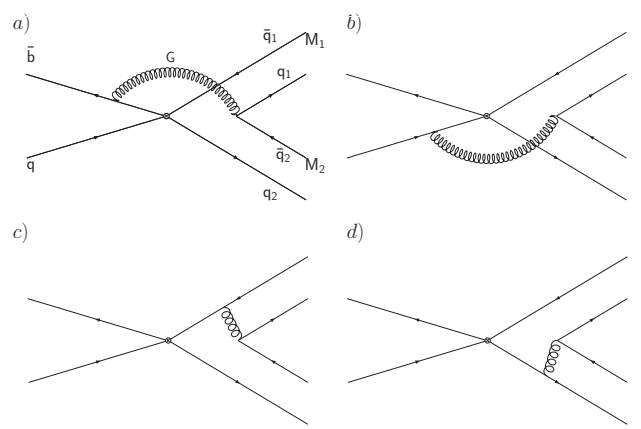

FIGURE 1. Feynman diagrams contributing to the amplitude of annihilation decays of $B$ mesons.

where $Z\left(p^{2}\right)$ is the Lorentz scalar quark wave function renormalization and $M\left(p^{2}\right)$ is the quark dynamical mass. At large momenta, and in the chiral limit, it is known that that the dynamical mass is related to the quark condensate $(<\bar{q} q>)$ as $M\left(p^{2}\right) \propto<\bar{q} q>/ p^{2}[6]$. The many estimates of the dynamical quark mass give values between $250 \mathrm{MeV}$ and 300 $\mathrm{MeV}[6,7]$. Here, since the mass function has a plateau at low momenta and decreases (in the chiral limit) very fast at high momenta [7], we can safely assume $Z\left(p^{2}\right) \approx 1$ and $S(p)=1 /(\iota \not p+M(0))$.

\section{ANNIHILATION DECAYS}

We compute the amplitudes for pure annihilation decays in the collinear approximation of pQCD at twist-3 approximation. This type of process occurs at $\alpha_{s}$ order in perturbative QCD. A typical annihilation decay of a B meson is shown in Fig. 1, where the bottom quark decays through the $\mathrm{W}$ exchange or via a penguin process and a quark-antiquark pair is created in the final state through a gluon emission by any of the quarks involved in the process. The channels with these characteristics that we shall analyze are the following charmless channels: $B_{s}^{0} \rightarrow \pi^{+} \pi^{-}, \pi^{0} \pi^{0}, B_{d}^{0} \rightarrow K^{+} K^{-}$; and charmed channels: $B^{0} \rightarrow D_{s}^{ \pm(*)} K^{\mp}, B^{+} \rightarrow D_{s}^{+(*)} \bar{K}^{0}, B_{s}^{0} \rightarrow D^{ \pm(*)} \pi^{\mp}, D^{0} \pi^{0}, \bar{D}^{0} \pi^{0}$.

The branching ratios of each decay is calculated from

$$
\mathscr{B}\left(B \rightarrow M_{1} M_{2}\right)=\frac{\tau_{B} p_{c}}{8 \pi m_{B}^{2}}\left|\mathscr{A}\left(B \rightarrow M_{1} M_{2}\right)\right|^{2},
$$

where $\tau_{B}$ is the $B$ meson lifetime, $p_{c}$ is the momentum of the final state particles in the $B$ meson rest frame.

We perform the numerical calculation considering the gluon mass scale with values $m_{g}=400 \mathrm{MeV}, 500 \mathrm{MeV}$. The dynamical masses of the light quarks are $M(0)=m_{u}=$ $m_{d}$, in the interval $250 \mathrm{MeV}<M(0)<300 \mathrm{MeV}$, and the same value is assumed for the $s$ quark. The Wilson's coefficients are computed using the equations given in the appendices of Ref. [9]. Other numerical parameters such as the masses, decay constants and life times of mesons, and the CKM parameters are taken from [10]. In Table 1 we show the results obtained and the experimental data available for each decay channel. 
TABLE 1. Branching ratios for $B$ decays for the gluon mass scale $m_{g}=400 \mathrm{MeV}$ and $500 \mathrm{MeV}$. The error corresponds to the variation of the dynamical quark mass, $250 \mathrm{MeV}<$ $m_{q}<300 \mathrm{MeV}$. The available experimental data are also shown.

\begin{tabular}{lrrr}
\hline Channel & $\mathscr{B}\left(m_{g}=400 \mathrm{MeV}\right)$ & $\mathscr{B}\left(m_{g}=500 \mathrm{MeV}\right)$ & Experimental data [10] \\
\hline$B^{0} \rightarrow K^{+} K^{-}$ & $(5.83 \pm 0.67) \times 10^{-8}$ & $(4.34 \pm 0.01) \times 10^{-8}$ & $<4.1 \times 10^{-7}$ \\
$B_{s}^{0} \rightarrow \pi^{+} \pi^{-}$ & $(11.08 \pm 1.06) \times 10^{-7}$ & $(5.65 \pm 0.27) \times 10^{-7}$ & $<1.7 \times 10^{-6}$ \\
$B_{s}^{0} \rightarrow \pi^{0} \pi^{0}$ & $(5.54 \pm 1.06) \times 10^{-7}$ & $(2.83 \pm 0.27) \times 10^{-7}$ & $<2.1 \times 10^{-6}$ \\
$B^{0} \rightarrow D_{s}^{-} K^{+}$ & $(5.67 \pm 0.73) \times 10^{-5}$ & $(2.84 \pm 0.16) \times 10^{-5}$ & $(2.9 \pm 0.5) \times 10^{-5}$ \\
$B^{0} \rightarrow D_{s}^{+} K^{-}$ & $(5.69 \pm 0.66) \times 10^{-8}$ & $\left(2.71 \pm 0.22 \times 10^{-8}\right.$ & \\
$B^{+} \rightarrow D_{s}^{+} \bar{K}^{0}$ & $(6.09 \pm 0.69) \times 10^{-8}$ & $(1.34 \pm 0.22) \times 10^{-8}$ & $<9 \times 10^{-4}$ \\
$B_{s}^{0} \rightarrow D^{-} \pi^{+}$ & $(2.41 \pm 0.05) \times 10^{-6}$ & $(1.65 \pm 0.55) \times 10^{-6}$ & \\
$B_{s}^{0} \rightarrow \bar{D}^{0} \pi^{0}$ & $(1.21 \pm 0.03) \times 10^{-6}$ & $(0.86 \pm 0.25) \times 10^{-6}$ & \\
$B_{s}^{0} \rightarrow D^{+} \pi^{-}$ & $(8.13 \pm 0.85) \times 10^{-7}$ & $(3.52 \pm 1.35) \times 10^{-7}$ & \\
$B_{s}^{0} \rightarrow D^{0} \pi^{0}$ & $(4.04 \pm 0.44) \times 10^{-7}$ & $(3.39 \pm 0.95) \times 10^{-7}$ & \\
$B^{0} \rightarrow D_{s}^{*-} K^{+}$ & $(2.35 \pm 0.15) \times 10^{-5}$ & $(1.56 \pm 0.05) \times 10^{-5}$ & $(2.2 \pm 0.6) \times 10^{-5}$ \\
$B^{0} \rightarrow D_{s}^{*+} K^{-}$ & $(5.55 \pm 0.41) \times 10^{-8}$ & $(2.21 \pm 0.06) \times 10^{-8}$ & \\
$B^{+} \rightarrow D^{*+} \bar{K}^{0}$ & $(5.51 \pm 0.43) \times 10^{-8}$ & $(2.36 \pm 0.06) \times 10^{-8}$ & $<9 \times 10^{-4}$ \\
$B_{s}^{0} \rightarrow D^{*-} \pi^{+}$ & $(3.36 \pm 0.06) \times 10^{-6}$ & $(1.88 \pm 0.21) \times 10^{-6}$ & \\
$B_{s}^{0} \rightarrow D^{*+} \pi^{-}$ & $(3.26 \pm 0.29) \times 10^{-8}$ & $(3.91 \pm 1.31) \times 10^{-8}$ & \\
\hline
\end{tabular}

In conclusion, we have studied some decay channels of $B_{s}^{0}, B_{d}^{0}, B^{+}$mesons which occur through the annihilation diagrams. We have argued that infrared finite gluon propagators and running coupling constants obtained as solutions of the QCD SchwingerDyson equations, may serve as a natural cutoff for the end-point divergences that appear in the calculation of these decays. Also, we use the constituent quark mass for the light quarks in the propagators to be able to perform the twist-3 calculation of the amplitudes. Comparing to the data available, our calculations are very satisfactory, notably for the two channels with actual measurements $B^{0} \rightarrow D_{s}^{-} K^{+}$and $B^{0} \rightarrow D_{s}^{-*} K^{+}$.

\section{ACKNOWLEDGMENTS}

This research was supported by the FAPESP (CMZ) and CNPq (AAN).

\section{REFERENCES}

1. G. P. Lepage and S. J. Brodsky, Phys. Rev. D 22, 2157 (1980); A. Szczepaniak, E. M. Henley and S. J. Brodsky, Phys. Lett. B 243, 287 (1990).

2. M. Beneke, G. Buchalla, M. Neubert and C. T. Sachrajda, Nucl. Phys. B 606, 245 (2001)

3. H. n. Li and H. L. Yu, Phys. Rev. Lett. 74, 4388 (1995).

4. J. M. Cornwall, Phys. Rev. D 26, 1453 (1982).

5. A. A. Natale and C. M. Zanetti, Int. J. Mod. Phys. A 24, 4133 (2009).

6. C. D. Roberts and A. G. Williams, Prog. Part. Nucl. Phys. 33, 477 (1994); C. D. Roberts, Prog. Part. Nucl. Phys. 61, 50 (2008).

7. P. Maris and C. D. Roberts, [nucl-th/9710062].

8. G. Buchalla, A. J. Buras and M. E. Lautenbacher, Rev. Mod. Phys. 68, 1125 (1996).

9. C. D. Lu, K. Ukai and M. Z. Yang, Phys. Rev. D 63, 074009 (2001)

10. C. Amsler et al. [Particle Data Group], Phys. Lett. B 667, 1 (2008). 
Copyright of AIP Conference Proceedings is the property of American Institute of Physics and its content may not be copied or emailed to multiple sites or posted to a listserv without the copyright holder's express written permission. However, users may print, download, or email articles for individual use. 